\title{
Metamers in the haptic perception of heaviness and moveableness
}

\author{
KEVIN SHOCKLEY \\ University of Cincinnati, Cincinnati, Ohio \\ and \\ CLAUDIA CARELLO and M. T. TURVEY \\ University of Connecticut, Storrs, Connecticut
}

\begin{abstract}
It is hypothesized that heaviness perception for a freely wielded nonvisible object can be mapped to a point in a three-dimensional heaviness space. The three dimensions are mass, the volume of the inertia ellipsoid, and the symmetry of the inertia ellipsoid. Within this space, particular combinations yield heaviness metamers (objects of different mass that feel equally heavy), whereas other combinations yield analogues to the size-weight illusion (objects of the same mass that feel unequally heavy). Evidence for the two types of combinations was provided by experiments in which participants wielded occluded hand-held objects and estimated the heaviness of the objects relative to a standard. Further experiments with similar procedures showed that metamers of heaviness were metamers of moveableness but not metamers of length. A promising conjecture is that the haptic perceptual system maps the combination of an object's inertia for translation and inertia for rotation to a perception of the object's maneuverability.
\end{abstract}

In two important respects, scientific psychology began with inquiry into the perception of weight - specifically, into the question of how a person's impression of heaviness relates to the weight of an object that is either passively supported or lifted (Weber, 1834/1978). The main outcome of Weber's original work was the understanding that the relation of heaviness to weight was imperfect but systematic. Discrimination among objects that differed in weight was accommodated by a simple constant ratio: the minimal difference needed to distinguish the weight of a comparison object from that of a standard object, divided by the weight of the standard object. The secondary outcome of Weber's original work was that the discrimination of weight was greater (i.e., the constant was smaller) when objects were lifted rather than passively supported. The superiority of lifting pointed to the involvement of the mechanoreceptors embedded in muscles and in the attachments of muscles to tendons. The collective perceptual function of these mechanoreceptors was referred to as muscle sense by Bell (1826) and as dynamic touch by Gibson (1966).

\section{Nonuniqueness of Weight Perception}

The limits on weight discrimination observed by Weber did not fully express the imperfect mapping between weight and its perception. Charpentier (1891) found that for two

This research was supported by NSF Grant SBR 00-04097. Correspondence should be addressed to K. Shockley, Department of Psychology, Mail Location 0376, University of Cincinnati, Cincinnati, OH 45221-0376 (e-mail: kevin.shockley@uc.edu). spheres of equal mass but of different diameters ( $40 \mathrm{~mm}$ vs. $100 \mathrm{~mm}$ ), the smaller diameter sphere was perceived to be heavier. An intuitively plausible interpretation is that the sight of a larger object elicits the application of greater initial force than does the sight of a smaller object, with the resulting faster lifting motion giving rise to the impression that the larger object is lighter (e.g., Granit, 1972; H. E. Ross, 1969). Despite evidence consistent with the preceding vision-based account of Charpentier's discovery (e.g., Davis \& Roberts, 1976; Gordon, Forssberg, Johansson, \& Westling, 1991; Kingma, Salversbergh, \& Tousaint, 1999; Payne \& Davis, 1940), it proves to be the case that vision, though sufficient, is not necessary (Ellis \& Lederman, 1993; see also Amazeen, 1997, 1999). The effect of size on heaviness is evident in conditions of strictly haptic perception.

The nonuniqueness of weight perception (with or without a contribution of vision) has encouraged some explanations in terms of mental or neural states (Jones, 1986). The fact that a single physical weight gives rise to many perceived weights implies that the determination of perceived weight is an achievement of the nervous system, and not of objective physical constraints. Information integration models (Anderson, 1970; Gregory, 1974; Sjöberg, 1969) and expectation models (Davis \& Brickett, 1977; Davis \& Roberts, 1976; H. E. Ross \& Gregory, 1970), for example, both implicate internal comparisons as arbiters of the resultant perception of weight. These explanations are in the spirit of those that have long characterized color perception (albeit where the implicated retinal physiology has been well articulated). Some have gone so far as to label this tack a kind of subjectivism (e.g., Clark, 2000; Dretske, 
1995; Hardin, 1993; Hilbert, 1992; P. W. Ross, 2001). Consider a typical characterization of color perception:

The results described here. . . suggest that the nervous system, rather than analyze colours, takes what information there is in the external environment, namely, the reflectance of different surfaces for different wavelengths of light, and transforms that information to construct colours, using its own algorithms to do so. In other words, it constructs something which is a property of the brain, not the world outside (Zeki, 1983, p. 764).

As a parallel of color subjectivism, weight subjectivism is discomfiting in its logical entailments. Most notably, if perceived weight is a construct, then does it not require that weight-like color - should be considered a property of the brain, and not of objects in the world outside (cf. Turvey, Whitmyer, \& Shockley, 2001)? Although most investigators of weight perception may not accept the inevitability of this slippery slope, it is not easily avoidable.

As an alternative, the nonuniqueness of weight perception might be taken to encourage a reexamination of the weight-perception problem. When a person remarks on the heaviness of an object, how should the object be characterized physically? Furthermore, when a person remarks on the heaviness of an object, is "heaviness" to be taken at face value as the proper characterization of the perception? These questions are made all the more compelling by the fact that the one-to-many mapping between weight and perceived weight is only one side of the coin. Inspection of the data sets from experiments that involved multiple combinations of weight and size (e.g., Cross \& Rotkin, 1975) reveals a many-to-one mapping: Objects with different weight values are perceived as having the same weight. Again, a parallel with color perception is useful: Many combinations of physical properties give rise to the same color experience. In color vision, these equivalents are called metamers. Below, we will see how we might configure their haptic counterparts. Before we do so, it is important to put the generalization of the metamer notion into perspective.

\section{Metamer Hierarchy}

In an inquiry into Bloch's law (tradeoff in vision between intensity and duration with respect to threshold-detection performance), Loftus and Ruthruff (1994) highlighted the idea that metamerism can be of more than one form and of more than one origin. As Backus (2002) has suggested, Loftus and Ruthruff hinted at the possibility of a hierarchy of metamers with levels defined by the descriptions under which two physically different stimuli were indistinguishable. Two or more stimuli with the same value of duration $X$ intensity are only perceptual metamers (manifest at the time of viewing) up to a limiting duration, but they can be memory metamers (equivalent to recall performance subsequent to viewing) at any duration. For the sensoryresponse functions of equal-product stimuli, sameness in shape yields perceptual metamers, and sameness in area yields memory metamers (Loftus \& Ruthruff, 1994).
In Loftus and Ruthruff's (1994) terms, two physically different stimuli are metameric if they produce identical responses at some stage of the perceptual-cognitive system (see also Sekuler \& Blake, 1994). In the case of traditional color metamers, identical responses are presumed to occur at the transduction stage. It is perhaps useful to refer to them as sensory metamers. Although the distinction may be difficult to make consistently, Backus (2002) has proposed that the contrast between sensory and perceptual metamers is a contrast between data "lost" at peripheral and central sites, respectively, during transduction and computational processes, respectively. At a given vergence eye posture, it is possible to create distinct stereoscopic disparity patterns that are perceptually indistinguishable with respect to slant. However, changing vergence without changing the stereoscopic disparity patterns renders the stimuli nonmetameric. Arguably, stereoscopic slant metamers arise because the distinguishing physical horizontal disparity is lost when entered into computations (e.g., involving vergence) that interpret the disparity as depth (Backus, 2002). The preceding outcome and argument are in accord with Sekuler and Blake's (1994) claim that much can be learned about vision, and presumably about perception in general, by determining which stimuli are metamers and the conditions under which they remain so.

The vision literature is a source of other metamers that seem to fit the perceptual rather than sensory definitionnamely, texture (Richards \& Riley, 1977), speed of motion (e.g., Festa \& Welch, 1998) and direction of motion (e.g., Treue, Hol, \& Rauber, 2000; Williams, Tweten, \& Sekuler, 1991). Direction metamers for overlays of moving transparent patterns have been attributed to neural processes at the level of the extrastriate cortex (Treue et al., 2000). As with stereoscopic slant metamers, motion direction metamers can be rendered distinguishable (nonmetameric) by a specific change of circumstances. Simply, the lost individual and distinct direction-components constituting metameric stimuli can be recovered, as it were, by the addition of disparity differences to the motion stimuli. Treue et al. (2000) conjecture that the neural population-coding scheme they propose to accommodate perceived visual motion direction is so simple and general that it could be a source of metameric experiences in other perceptual systems.

In summary, the lesson of contemporary research in vision is that there are different kinds of metamers with different theoretical implications. For some metamers, for example, stereoscopic slant and motion direction metamers, the metamer-breaking operations are (or seem to be) changes in stimulus information (e.g., about object depth). Likewise, the distance of the observer can be a metamerbreaking operation. Two photographs of the same object that are physically distinguished by the presence of higher spatial frequencies in one photograph but not the other are metameric when viewed from a distance (higher frequencies are outside acuity limits) but nonmetameric when viewed up close (Sekuler \& Blake, 1994). For other metamers - for example, those of color - the metamer- 
breaking operations are changes in stimulus energy (e.g., from incandescent light to daylight). As noted, the former metamers can be labeled perceptual and the latter sensory (Backus, 2002).

To preview the present research, it may be necessary to refine the metamer hierarchy to include varieties of cognitive metamers in addition to those of memory. Wielded nonvisible objects that are metamers under one perceptual goal ("how heavy?") are not metamers under a different perceptual goal ("how long?"). Like memory metamers, these stimuli are distinguishable in perception - they do not feel identical in all respects. But they do feel the same with respect to the focal property. The metamer-breaking operation, then, changes the intention to perceive one property as opposed to another. This extension of the hierarchy is consonant with Sekuler and Blake's (1994, p. 172) axiom that "any condition that alters the response of the nervous system influences whether stimuli are metamers." Certain perceptual metamers are likely to be intendedproperty metamers as well (e.g., metameric with respect to loudness but not to pitch; metameric to surface slant but not to surface texture). However, the question of the perceiver's intent is typically not posed of simple, justdetectable stimuli for which the conditions of presentation effectively preclude exploration. For example, stereoscopic slant metamers do not persist with saccades (Backus, 2002). Several features make intentionally defined cognitive metamers in touch an especially compelling elaboration of the metamer hierarchy. First, whole three-dimensional objects are presented. Second, exploration is not limited, either in time or in wielding style (as long as the object remains firmly grasped in one hand). Finally, a single energy array - the deformation of the tissues of the hand and arm - is rendered informative about different properties by the perceiver's intention. We will now turn to this energy array in some detail.

\section{Three-Dimensional Heaviness Space}

Everyday interactions with manually grasped objects (e.g., wielding, hefting, lifting, and carrying books, mugs, forks, etc.) typically consist of translations and rotations. These interactions combine forces proportional to an object's resistance to translational acceleration (its mass) and torques scaled to an object's resistance to rotational acceleration (its inertia tensor). Research on the wielding of nonvisible hand-held objects suggests that both the object's mass and its inertia tensor determine how heavy the wielded object feels. In particular, a three-dimensional heaviness space is suggested by experiments in which the relevant (factorial) manipulations of the inertia tensor are the volume and symmetry of its corresponding inertial ellipsoid (Shockley, Grocki, Carello, \& Turvey, 2001; Turvey, Shockley, \& Carello, 1999). ${ }^{1}$ The quantities ellipsoid volume and ellipsoid symmetry can be understood as important to controlling the level and patterning of muscular forces, respectively. Ellipsoid volume expresses the absolute magnitudes of the principal moments of the object's inertia tensor. It is defined as ellipsoid volume $=4 \pi / 3\left(I_{1} \times I_{2} \times\right.$
$\left.I_{3}\right)^{-1 / 2}\left(\right.$ where $_{1}, 2$, and ${ }_{3}$ index the maximum, intermediate, and minimum principal moments or eigenvalues of the inertia tensor, respectively). Ellipsoid symmetry expresses the relative magnitudes of the principal moments. It can be usefully defined as ellipsoid symmetry $=2 I_{3} /\left(I_{1}+I_{2}\right)$ (Shockley et al., 2001; Turvey et al., 1999).

The hypothesized heaviness space mass $\times$ ellipsoid volume $\times$ ellipsoid symmetry is depicted in Figure 1 . Presumably, there is a haptic mapping $\Psi$ that relates each point in this space to a heaviness perception. An approximation to heaviness perception $=\Psi$ (mass, ellipsoid volume, ellipsoid symmetry) was derived from experiments in which levels of mass, ellipsoid volume, and ellipsoid symmetry were independently manipulated (Shockley et al., 2001). Combinations of mass, ellipsoid volume, and ellipsoid symmetry that correspond to the same value of perceived heaviness are shown as planes in Figure 1. These planes of equal heaviness may be viewed as physical conditions that define heaviness metamers. By analogy with color metamers, two physical objects with different mass, ellipsoid volume, and ellipsoid symmetry can be perceived as equally heavy as long as the inertial properties of each object are combined in the right proportions. Furthermore, any two points in different equal-heaviness planes that share the same mass coordinate may be viewed as physical conditions that yield an analogue of the "size-weight illusion." Despite the sameness in weight, the two objects corresponding to these two points (see Figure 1) should be perceived to differ in heaviness.

\section{Tensor Objects}

Appropriate manipulations of mass, ellipsoid volume, and ellipsoid symmetry can be achieved through the use of tensor objects (Amazeen \& Turvey, 1996), examples of which are shown in Figure 2. Tensor objects consist of five rods of fixed mass and linear dimensions and a variable number of attached metal rings. One rod is the stem. The other rods are branches attached to the stem so as to form two cross-pieces perpendicular to the stem and to each other. The branches are attached through a hub that can be positioned freely along the length of the stem. The total mass of a tensor object can be prescribed by simply selecting particular magnitudes of the masses of the attached metal rings. Specific inertia tensors relative to $O$ (the rotation point in the wrist when the stem of a tensor object is grasped at one end; see Figure 2) can be prescribed by selecting specific positions of the hub and/or specific positions and mass magnitudes of the metal rings attached to the branches and the stem. ${ }^{2}$

\section{Present Experiments}

In five experiments, we used tensor objects to examine the space depicted in Figure 1. Experiments 1, 2, and 3 required a judgment of heaviness while objects were wielded freely. Experiment 4 required a judgment of moveableness. More specifically, Experiment 4 posed the question of whether, in general, the haptic perception of an object's heaviness is equally understandable as the perception of 


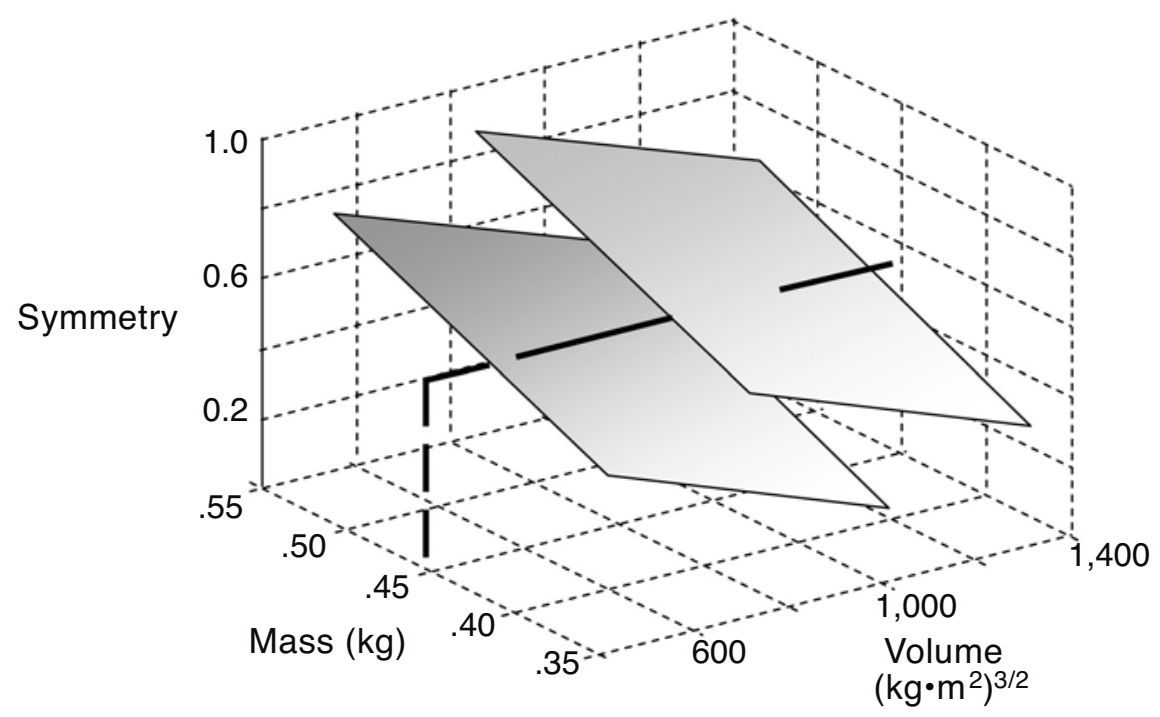

Figure 1. Heaviness/moveableness space consisting of mass and the volume and symmetry of the inertia ellipsoid. Each point in a plane identifies a combination of the designated variables that yields the same heaviness/moveableness percept as the combination of variables corresponding to any other point in the plane. Parallel planes reflect different sets of metameric matches. The solid line intersecting more than one plane illustrates that a single mass (designated as $.45 \mathrm{~kg}$ ) may be perceived differently depending on the inertia tensor variables (a variant of the size-weight illusion). The lower plane represents heavier metamers (heaviness decreases with increases in both ellipsoid symmetry and ellipsoid volume).

an object's resistance to being moved - the perception of its maneuverability or controllability (Shockley et al., 2001). A fifth and final experiment addressed whether the metamers of Figure 1 might emerge, whatever the required judgment. A haptic judgment was required that, unlike heaviness or moveableness, does not necessarily refer to demands on the movement system. Accordingly, in Experiment 5, participants judged object length.

\section{EXPERIMENT 1}

As noted, in the heaviness space of Figure 1, a mass can be associated with two different pairings of ellipsoid volume and ellipsoid symmetry that correspond to points lying in two different equal-heaviness planes. Because these two points identify different heaviness perceptions for an object of a given weight, they define a variant of the size-weight illusion.

Experiment 1 was a special version of Charpentier's (1891) experiment. A participant wielded, in succession, two objects with the task of judging which was heavier. In contrast to Charpentier's objects, the objects of Experiment 1 were equal in both weight and size, differing only in the second moments (inertia tensor) of their mass distributions. Furthermore, in contrast to Charpentier's participants, the participants in Experiment 1 were prohibited from seeing the two objects.

The objects in question were two tensor objects, each weighing $.45 \mathrm{~kg}$. They were constructed according to the regression equation from Shockley et al. (2001) in which mass, ellipsoid volume, and ellipsoid symmetry had been manipulated factorially - namely, perceived heaviness $=$ $112.94+147.38$ mass -0.03 ellipsoid volume -62.76 ellipsoid symmetry. ${ }^{3}$ Specifically, the ellipsoid volumes and symmetries were chosen to render, for the given mass value, two distinct values of perceived heaviness. It was expected that the participants' choice as to the heavier object would reflect this distinction.

\section{Method}

Participants. Ten undergraduates at the University of Connecticut participated in partial fulfillment of a course requirement. (All participants in the present and subsequent experiments were informed as to the nature of the experiment, and all gave their consent to participate.)

Materials. Two tensor objects of $.45 \mathrm{~kg}$ were used as test objects (for comparison, a coffee mug weighs $.27 \mathrm{~kg}$, a can of soup weighs $.42 \mathrm{~kg}$, and a textbook weighs $.80 \mathrm{~g}) .{ }^{4}$ They were equal in the linear dimensions $($ stem $=.45 \mathrm{~m}$, each arm $=.40 \mathrm{~m})$ and radii (inner $=$ $.006 \mathrm{~m}$, outer $=.009 \mathrm{~m}$ ) of the rods and distinguished only by the positions of the attached metal rings on the rods perpendicular to the stem (Figure 2). The ellipsoid volume and ellipsoid symmetry were, respectively, 2,210 and .49 for Object 1 and 588 and .79 for Object 2 . As depicted in Figure 2, the attached metal rings were close to the hub for Object 1 and far from the hub for Object 2, with the hubs of both objects at the same location along their stems. According to the regression equation based on the factorial experiment of Shockley et al. (2001; see above), the predicted heaviness of Object 2 was greater than that of Object 1.

Procedure. The two tensor objects were never seen in the experiment (the participants were blindfolded) and no information was given about their designs. Only one object was wielded at a time. The object, placed into the participant's right hand by the experimenter, 

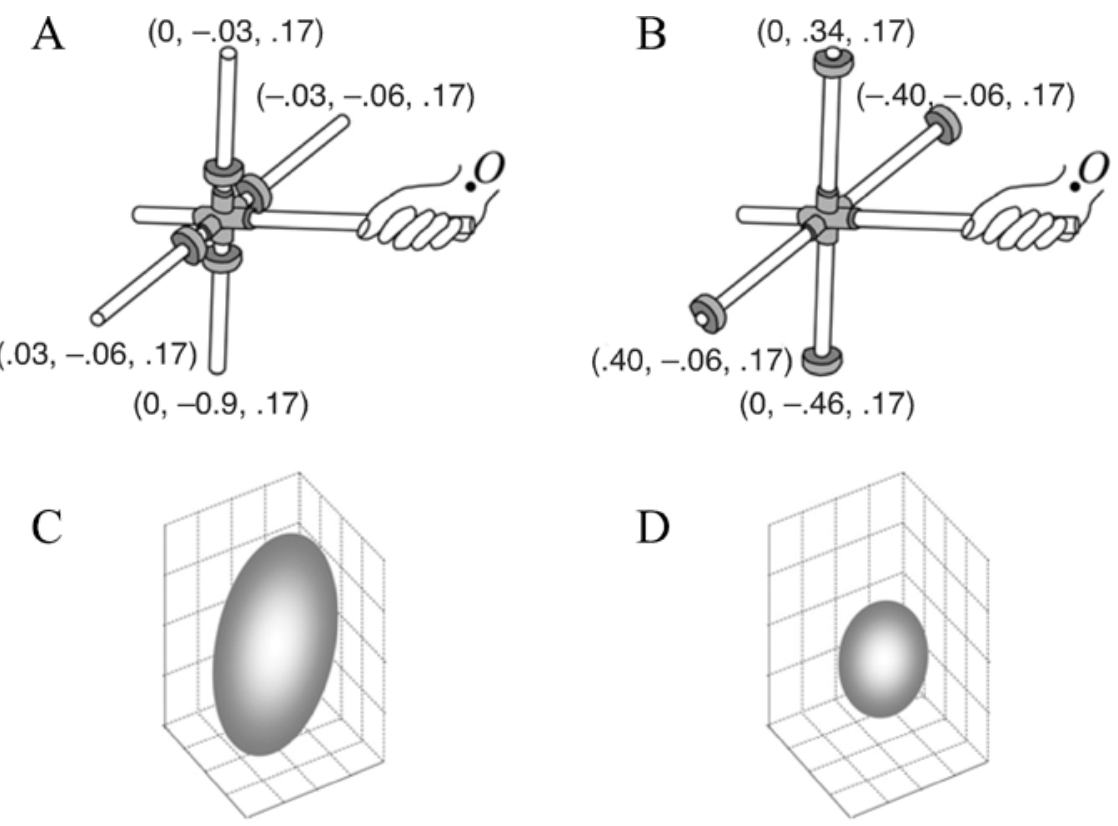

$\mathrm{D}$

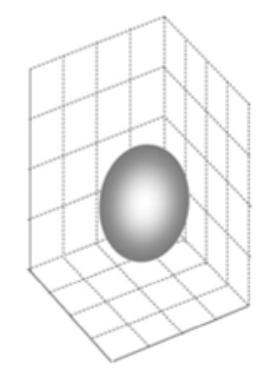

E

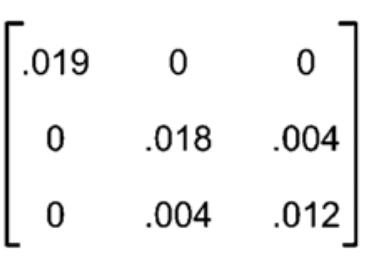

$\mathrm{F}\left[\begin{array}{ccc}.035 & 0 & 0 \\ 0 & .033 & .004 \\ 0 & .004 & .044\end{array}\right]$

$\mathrm{G}\left[\begin{array}{ccc}.020 & 0 & 0 \\ 0 & .019 & 0 \\ 0 & 0 & .010\end{array}\right]$

$\mathrm{H}\left[\begin{array}{ccc}.045 & 0 & 0 \\ 0 & .035 & 0 \\ 0 & 0 & .032\end{array}\right]$

Figure 2. Panels $A$ and $B$ depict the two tensor objects of Experiment 1 with the $x, y$, and $z$ coordinates of the hub and the attached metal rings. In general, the cross bars of a tensor object are moveable as a unit, and the metal rings attached to them can be varied in mass and distance from the hub. These variations permit the construction of particular inertia tensors relative to the origin of rotation axes at $O$. Panels $C$ and $D$ depict the corresponding inertia ellipsoids. The ellipsoids differ in size, shape, and orientation. For each object, the $3 \times 3$ matrices are the corresponding inertia tensors computed in the spatial coordinate system $O_{x y z}$ $(E$ and $F$ ) and its diagonalized form computed about the principal directions or eigenvectors ( $G$ and $H)$. The orientation difference between the two ellipsoids is due to the fact that the maximum principal moment is more closely related to rotation about the $z$ axis in the case of the object depicted on the right and about the $y$ axis in the case of the object depicted on the left.

was grasped firmly by the participant with the proximal end of the object's stem flush with the bottom of the fist and with the stem always parallel to the fist (see Figure 2). Wielding was by rotations of the hand in three dimensions with the forearm supported. The participants were free to wield for as long as needed and to elect whatever pattern and vigor of wielding they wished. ${ }^{5}$ On a trial, the participants wielded one object, then the other, with the number of alternations dictated by the participants' level of confidence in the heaviness distinction. There were 10 trials, with the order of the two objects randomized across trials. (This procedure and the similar procedures of Experiments 2-5 were approved by the Institutional Review Board of the University of Connecticut.)

\section{Results and Discussion}

For the 10 participants, the number of times out of 10 that Object 2 was chosen as the heavier object was 9, 2, 7, $7,8,9,7,9,9$, and 10. Expressed as probabilities, these choices were compared to the chance value of .5 in a onesample, one-tailed $t$ test $[t(9)=3.77, p<.003]$. 
Experiment 1 was an abstract version of the classical demonstration of the size-weight illusion. It was "abstract" in the sense that the size difference between two objects of equal weight was encoded as a difference in the second moments of their mass distributions. In terms of linear dimensions (length and width), the two objects were of equal size. With the exception of one participant, the object predicted to be heavier on the basis of Figure 1 was perceived as heavier. This outcome corroborated the previous demonstrations of the abstracted size-weight illusion with tensor objects reported by Amazeen and Turvey (1996), Turvey et al. (1999), and Shockley et al. (2001).

\section{EXPERIMENT 2}

Experiment 2 extended the investigation of the haptic analogue of the size-weight illusion in the context of the heaviness space of Figure 1. Consider two values of mass, $X$ and $Y$. Suppose that $X$ is associated with two combinations of ellipsoid volume and ellipsoid symmetry to produce two values of perceived heaviness separated by the magnitude $\Delta$. Then, from the parallelism of equal-heaviness planes, it ought to be possible to choose for $Y$ two combinations of ellipsoid volume and ellipsoid symmetry that produce two different values of perceived heaviness that are separated by the same magnitude $\Delta$. Experiment 2 was a test of the preceding expectation.

Table 1 identifies two sets, each containing two tensor objects constructed according to the regression equation from Shockley et al. (2001). Between sets, the objects differed in mass. Within a set, the objects were identical in mass and different in ellipsoid volume and ellipsoid symmetry (corresponding to two points in different equalheaviness planes). The volume and symmetry values were chosen so that the two objects at one mass level would differ in perceived heaviness to the same degree as the two objects at the other mass level. It was predicted that perceived heaviness would be affected significantly by both mass and objects within a set but that the two factors would not interact.

\section{Method}

Participants. Eighteen undergraduates at the University of Connecticut participated in partial fulfillment of a course requirement.

Materials. At each of two levels of mass $(.37$ and $.53 \mathrm{~kg}$ ), two tensor objects were configured based on the aforementioned regres- sion equation. The ellipsoid volume and ellipsoid symmetry of the two tensor objects at each mass level (Table 1) were designed such that Object 2 would be perceived as heavier than Object 1, Object 4 would be perceived as heavier than Object 3, and the Object 2Object 1 difference would be equal to the Object 4 - Object 3 difference. A fifth object served as the standard for magnitude estimations. It was the same as the standard object of Shockley et al. (2001). As in Experiment 1, the objects of equal mass were also equal in the lengths and radii of the rods and in the location of the hub on the stem. They were distinguished only by the positions of the attached metal rings on the rods perpendicular to the stem (see Figure 2).

Procedure. The basic procedure was the same as in Experiment 1 with the exception that perceived heaviness was reported by magnitude estimation relative to the standard. The standard, which was assigned a value of 100 , was wielded on every trial prior to wielding the test object for that trial. Participants were instructed that if an object felt twice as heavy as the standard, a report of 200 was appropriate; if an object felt half as heavy, a report of 50 was appropriate. Object presentations were randomized with five trials per object.

\section{Results and Discussion}

Perceived heaviness averaged over trials and participants is shown in Figure 3A. The predictions that Object 2 would be perceived as heavier than Object 1 and Object 4 would be perceived as heavier than Object 3 , and the Object 2 - Object 1 difference would be equal to the $\mathrm{Ob}$ ject 4 - Object 3 difference were evaluated using two within-subjects $t$ tests. A $t$ test on the difference scores between the two objects for each mass level (magnitude estimation for Object $2-$ Object 1 vs. magnitude estimation for Object 4 - magnitude estimation for Object 3 ) revealed no significant difference across mass levels $(p>$ $.05)$. A $t$ test on the sums of the two objects within a mass level (Object 1 + Object 2 vs. Object 3 + Object 4) revealed a significant effect of mass level on perception of heaviness $[t(18)=14.00, p<.0001]$. In agreement with predictions from the heaviness space, perceived heaviness was different for the two sets, and objects weighing $.37 \mathrm{~kg}$ (Objects 1 and 2) differed in perceived heaviness to the same degree as objects weighing .53 kg (Objects 3 and 4). As in the previous experiment, the expected pattern was obtained for the majority of participants: 14 of the 18 participants for the $.37-\mathrm{kg}$ objects and 12 of the 18 participants for the $.53-\mathrm{kg}$ objects. Only 2 people reversed the expected heaviness for each object. The expected and observed additive effect of mass and the ellipsoid volumeellipsoid symmetry combination is in agreement with previous theorizing and research suggesting independent

Table 1

Mass, Symmetry $(S)$, Volume $(V)$, and Eigenvalues $\left(I_{1}, I_{2}, I_{3}\right)$ of Tensor Objects in Experiment 2

\begin{tabular}{ccccccc}
\hline Object & $\begin{array}{c}\text { Mass } \\
(\mathrm{kg})\end{array}$ & $S$ & $\begin{array}{c}V \\
\left(\mathrm{~kg} \cdot \mathrm{m}^{2}\right)^{-3 / 2}\end{array}$ & $\begin{array}{c}I_{1} \\
\left(\mathrm{~kg} \cdot \mathrm{m}^{2}\right)\end{array}$ & $\begin{array}{c}I_{2} \\
\left(\mathrm{~kg} \cdot \mathrm{m}^{2}\right)\end{array}$ & $\begin{array}{c}I_{3} \\
\left(\mathrm{~kg} \cdot \mathrm{m}^{2}\right)\end{array}$ \\
\hline 1 & .374 & .576 & 2,340 & $.018 \dagger$ & .017 & .010 \\
2 & .374 & .793 & 940 & .032 & .026 & .023 \\
3 & .534 & .459 & 2,030 & .021 & .021 & .010 \\
4 & .534 & .783 & 420 & .058 & .043 & .040 \\
$5 *$ & .454 & .603 & 1,430 & .025 & .024 & .015 \\
\hline
\end{tabular}

*Object 5 , which was not used as a test object, served as the standard (with an assigned value of 100) for magnitude estimations. †Eigenvalues are reported to three significant digits. Calculations of $S$ and $V$ used all significant digits of $I_{1}, I_{2}, I_{3}$. 
A

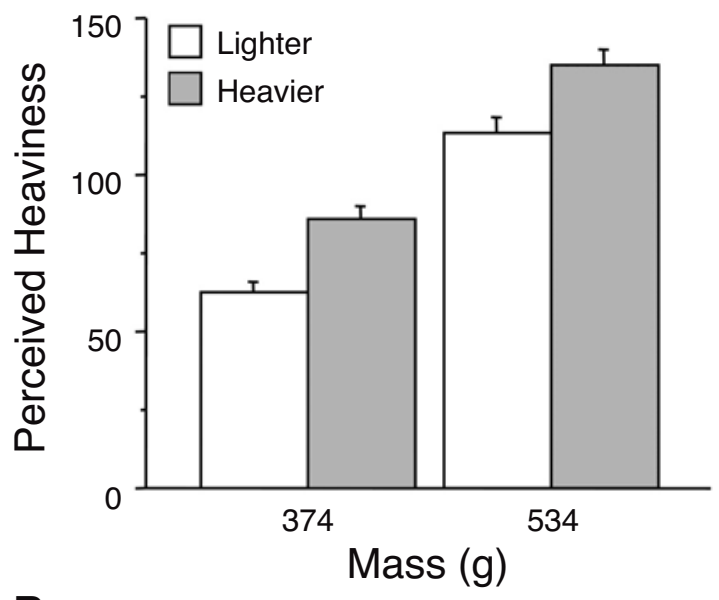

B

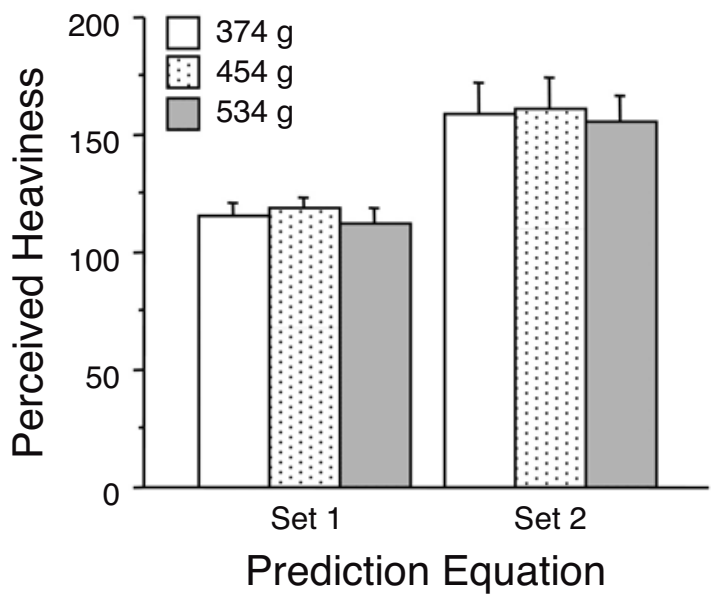

Figure 3. Mean perceived heaviness (with standard error bars) for Experiment 2 (A) and Experiment 3 (B).

haptic sensitivities to the inertia for translation and the inertia for rotation (Amazeen, 1997, 1999; Kreidfeldt \& Chuang, 1979; Shockley et al., 2001; Turvey et al., 1999).

The results of Experiment 2 together with those of Experiment 1 also affirm that variation of the second moment is sufficient, and that variation of the first moment is unnecessary, to produce variation in perceived heaviness. This is significant because it has been suggested that previous demonstrations of different heaviness judgments for objects of the same mass - that is, the same zeroth moment - could be due, in fact, to differences in the first moment that often accompany differences in the second moment (Kingma, Beek, \& van Dieen, 2002). In Experiment 1 , the two objects were equated on zeroth moment $(.45 \mathrm{~kg})$ and first moment $(.07 \mathrm{~kg} \cdot \mathrm{m})$ but felt different in heaviness, in accord with predictions from variation in the second moment. Experiment 2 replicated this pattern twice: Two objects of equal zeroth moment and equal first moment differed in perceived heaviness, whether the combination was $.37 \mathrm{~kg}$ and $.06 \mathrm{~kg} \cdot \mathrm{m}$ or $.53 \mathrm{~kg}$ and $.08 \mathrm{~kg} \cdot \mathrm{m}$. In both cases, perceived heaviness was in the direction predicted from consideration of the heaviness space of Figure 1 .

\section{EXPERIMENT 3}

In Experiment 3, we tested the heaviness metamers hypothesis. We produced two sets of three tensor objects. Each set included three values of mass $(.37, .45$, and $.53 \mathrm{~kg}$ ). Across the two sets, the values of ellipsoid volume and ellipsoid symmetry were chosen (by the foregoing regression equation) to render expected perceived heaviness the same within a set and different between sets. In summary, we predicted an effect of set, no effect of mass, and no mass $\times$ set interaction.

\section{Method}

Participants. Nine undergraduates at the University of Connecticut participated in partial fulfillment of a course requirement.

Materials. The details of the tensor objects are given in Table 2. Six of the objects were used as test objects. Objects 1-3 composed Set 1 (lower predicted perceived heaviness) and Objects 4-6 composed Set 2 (higher predicted perceived heaviness). Within a set, achieving the values of ellipsoid volume and ellipsoid symmetry required combined manipulations of the hub position and the positions of the metal rings on the rods perpendicular to the stem (see Figure 2). Because there were only two expected responses on average (two values of predicted perceived heaviness), two additional test objects were added to encourage response variation. The ninth object was the standard (Object 5 from Table 1). As in Experiment 2, this standard object was identical to that used in Shockley et al. (2001) in order to allow for predictions from their regression equation.

Procedure. The same procedure was used as in Experiment 2.

\section{Results and Discussion}

Mean perceived heaviness as a function of mass and set is shown in Figure 3B, where the pattern suggests heaviness metamers: Objects within a set yielded the same perceived heaviness. In confirmation, an ANOVA showed a main effect of set $[F(1,8)=16.83, p<.005]$ with no effect of mass $[F(2,16)=1.14, p>.05]$ and no mass $\times$ set interaction $(F<1)$.

The results of Experiments 1, 2, and 3 complement the results of research in which object mass and object size were manipulated (e.g., Cross \& Rotkin, 1975; Stevens \& Rubin, 1970). Physically distinct objects that were identical in mass were found to produce different magnitudes of perceived heaviness (as in the present Experiments 1 and 2 ), and physically distinct objects that differed in mass were found to produce the same magnitude of perceived heaviness (as in the present Experiment 3). In the three experiments, the objects were of one size (equal linear dimensions). Evidently, manipulations solely of mass distribution (here in the form of variations in the volume and symmetry of the inertial ellipsoid) can produce perceptual consequences analogous to manipulations of size.

\section{EXPERIMENT 4}

It has been argued that the proper question for the haptic perceptual system is "how moveable?" rather than "how heavy?" (Shockley et al., 2001; Turvey et al., 1999; 
Table 2

Mass, Symmetry $(S)$, Volume ( $V)$, and Eigenvalues $\left(I_{1}, I_{2}, I_{3}\right)$ of Tensor Objects in Experiments 3 and 4

\begin{tabular}{ccccccc}
\hline Object* & $\begin{array}{c}\text { Mass } \\
(\mathrm{kg})\end{array}$ & $S$ & $\begin{array}{c}V \\
\left(\mathrm{~kg} \cdot \mathrm{m}^{2}\right)^{-3 / 2}\end{array}$ & $\begin{array}{c}I_{1} \\
\left(\mathrm{~kg} \cdot \mathrm{m}^{2}\right)\end{array}$ & $\begin{array}{c}I_{2} \\
\left(\mathrm{~kg} \cdot \mathrm{m}^{2}\right)\end{array}$ & $\begin{array}{c}I_{3} \\
\left(\mathrm{~kg} \cdot \mathrm{m}^{2}\right)\end{array}$ \\
\hline 1 & .374 & 0.500 & 900 & $.035 \dagger$ & .035 & .018 \\
2 & .454 & 0.600 & 1,100 & .030 & .028 & .017 \\
3 & .534 & 0.736 & 1,200 & .028 & .023 & .019 \\
4 & .374 & 0.400 & 550 & .053 & .053 & .021 \\
5 & .454 & 0.551 & 620 & .044 & .043 & .024 \\
6 & .534 & 0.501 & 1,100 & .031 & .030 & .015 \\
7 & .374 & 0.619 & 2,510 & .017 & .016 & .010 \\
8 & .374 & 0.577 & 2,300 & .018 & .017 & .010 \\
\hline
\end{tabular}

*Objects 1-3 compose Set 1, and Objects 4-6 compose Set 2. Objects 7 and 8 were provided to extend the range of perceived weights. Object 5 from Table 1, which was not used as a test object, served as the standard (with an assigned value of 100) for magnitude estimations. $\dagger$ Eigenvalues are reported to three significant digits. Calculations of $S$ and $V$ used all significant digits of $I_{1}, I_{2}, I_{3}$.

Turvey et al., 2001). The question "how moveable?" is more general and reflects the haptic perceptual system's fundamental role in controlling the movements of limbs and hand-held objects. Consequently, participants' judgments of heaviness in Experiments 1-3 could well have been judgments of moveableness. Experiment 4 replicated Experiment 3 with the exception that participants reported how difficult the objects were to move, rather than how heavy the objects felt. It was expected that the metamers of Experiment 3 would be replicated in Experiment 4.

\section{Method}

Participants. Eight undergraduates at the University of Connecticut participated in partial fulfillment of a course requirement.

Materials. The details of the nine tensor objects used in Experiment 4 were the same as in Experiment 3 and are provided in Table 2.

Procedure. The same procedure was used as in Experiments 2 and 3 , with the exception that objects were evaluated with respect to how difficult they would be to move (relative to the same standard as in previous experiments). The standard was again assigned a value of 100 . The participants were instructed that if an object was twice as hard to move as the standard, a report of 200 was appropriate. If an object was half as hard to move, a report of 50 was appropriate (i.e., the greater the number, the more difficult the object was to move).

\section{Results and Discussion}

Mean moveableness perception as a function of mass and set is shown in Figure 4. In parallel with the analysis of heaviness, an ANOVA showed a main effect of set $[F(1,7)=275.26, p<.0001]$, with no effect of mass $[F(2,14)=1.44, p>.20]$ and no mass $\times$ set interaction $[F(2,14)=1.96, p>.15]$. An ANOVA conducted on the results of both Experiments 3 and 4 showed no effect of experiment $(F<1)$ and no interactions involving experiment $(F \mathrm{~s}<1)$. The only significant effect was that due to set $[F(1,15)=62.78, p<.0001]$. As is evident from inspection of Figures 3B and 4, the outcomes of the two experiments were identical. The minimal implication is that the questions "how heavy?" and "how moveable?" are equivalent; they are responded to in the same way.

\section{EXPERIMENT 5}

The parallel results in Experiments 3 and 4 allow the possibility that the metameric objects simply "feel the same" regardless of the property being judged. Perhaps there is nothing special about the question of moveableness. Perhaps the metamers of Experiment 3 would follow from any question about the wielded objects. A convergent manipulation is required to counter this possibility. An object's heaviness or moveableness implicates the capability of the participant's movement system in a way that an object's length does not. Accordingly, evidence in favor of an identity between heaviness and moveableness would be provided by a demonstration that reports of length do not yield the metameric matches of Experiment 4 . The present experiment was previewed in the introduction in the context of a metamer hierarchy. It may be interpreted

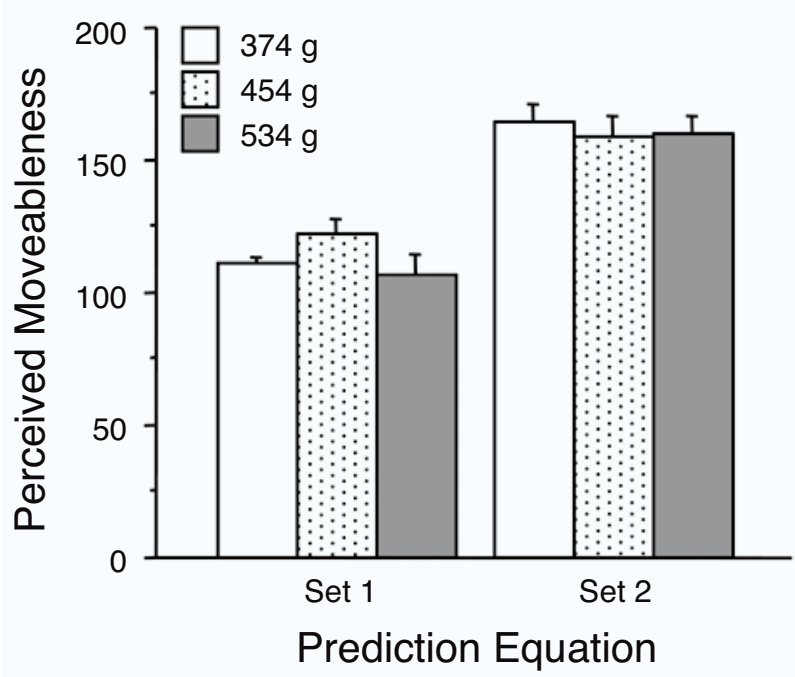

Figure 4. Mean perceived moveableness (with standard error bars) for Experiment 4. 
as an instance of a class of cognitive metamers in which objects that are identical under one intent are nonidentical under a different intent.

In Experiment 5, participants reported their impressions of how long the objects were, rather than their impressions of how heavy or how moveable the objects were. Previous research, using the complete identification design and associated statistical analyses (e.g., Ashby \& Townsend, 1986), has shown that length perception by wielding is independent from heaviness perception by wielding (Amazeen, 1997, 1999; see also Stroop, Turvey, Fitzpatrick, \& Carello, 2000). Moreover, expectations of relative perceived lengths can be calculated. It has been hypothesized that, in general, the perception of an object's largest linear extent varies as $I_{1}^{33} / I_{3}^{.25}$ (e.g., Turvey, Burton, Amazeen, Butwill, \& Carello, 1998). Consequently, two pairs of heaviness metamers were chosen (Objects 1 and 3 from Set 1, and Objects 4 and 6 from Set 2; see Table 2) so that the members of a pair differed with respect to the value of $I_{1}^{33} / I_{3}^{.25}: 0.91$ and 0.83 , respectively, for one pair, and 0.99 and 0.90 , respectively, for the other pair. Accordingly, if perceiving object length is distinct from perceiving object heaviness or moveableness, then the perceived length of Object 1 should be greater than that of Object 3, and the perceived length of Object 4 should be greater than that of Object 6 . That is, the metameric matches in Experiments 3 and 4 were not expected to be metameric matches in Experiment 5.

\section{Method}

Participants. Eight undergraduates at the University of Connecticut participated in partial fulfillment of a course requirement.

Materials. Referring to Table 2, Objects 1 and 3 composed Set 1 and Objects 4 and 6 composed Set 2 in this experiment.

Procedure. The same procedure was used as in the previous experiments, with the exception that objects were evaluated with respect to length (relative to the same standard as in previous experiments). The standard was again assigned a value of 100 . The participants were instructed that if an object felt twice as long as the standard, a report of 200 was appropriate. If an object was half as long, a report of 50 was appropriate.

\section{Results and Discussion}

Mean perceived length as a function of mass and set is shown in Figure 5. Unlike in Experiments 3 and 4, mass was significant $[F(1,7)=30.89, p<.001]$, meaning that the tensor objects composing Set 1 and Set 2 were not metamers. The significant effect of set $[F(1,7)=37.89$, $p<.001]$, and the nonsignificance of the mass $\times$ set interaction $[F(1,7)<1]$ conform to expectations from the $I_{1}^{33} / I_{3}^{25}$ constraint on perceived length. The mean value of $I_{1}^{33} / I_{3}^{2.25}$ for Set $2(0.95)$ was larger than that for Set 1 $(0.87)$ and the difference $(.09)$ between the larger and smaller ratios in Set 2 was approximately the same as the difference (.08) between the larger and smaller ratios in Set 1 .

As is evident from comparison of Figure 5 with Figures $3 \mathrm{~B}$ and 4, metamers in Experiment 4 (moveableness judgments) were metamers in Experiment 3 (heaviness

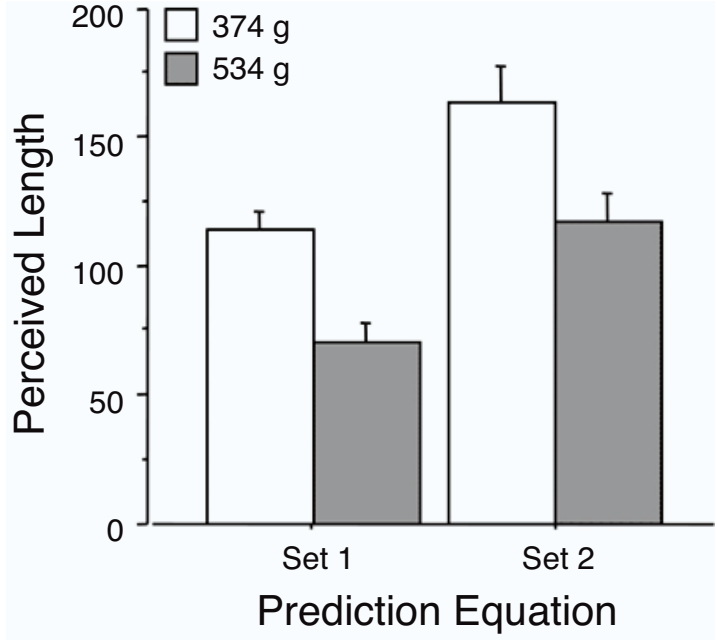

Figure 5. Mean perceived length (with standard error bars) for Experiment 5.

judgments) but were not metamers in Experiment 5 (length judgments). The contrast between Experiments 4 and 5 and the similarity between Experiments 3 and 4, lends support to the hypothesis that heaviness perception and moveableness perception are equivalent perceptions constrained by the mass distributions of objects in ways that differ from other perceptions achievable from wielding. In particular, the contrast provides encouragement for the view that when a person remarks on the heaviness of a nonvisible wielded or hefted object, the person is actually remarking on the object's disposition for being moved (Shockley et al., 2001; Turvey et al., 1999). In everyday interactions with objects, the forces and torques required to bring about motions in particular directions at particular speeds must be tailored to the resistances of any given object to translation and rotation. The disposition in question is not so much "moveable" as it is "maneuverable" or "controllable." However this particular disposition is defined, the claim that heaviness perception is actually moveableness perception implicates a special role for the haptic perceptual system. Given a hand-held object, the system must register the object's resistances to translation and rotation in a form and a manner suited to the requirements for generating the muscle tensile states needed to move the object in a desired way.

\section{GENERAL DISCUSSION}

The present research suggests that mass and the inertia tensor, when appropriately expressed in relation to the human motor system, may define a space that constrains the haptic perception of an object's disposition to be moved. Particular conjunctions of these parameters of the rigid body laws, as represented in Figure 1, define objects of unequal weight that are equally moveable. Other conjunctions define objects of equal weight that are unequally 
moveable. Conjunctions of the first kind define metamers (of moveableness); conjunctions of the second kind define nonmetamers that are perceptual equivalents of the sizeweight illusion.

The development of the metamer concept in the present sequence of experiments is consistent with the view of metamers advanced by Sekuler and Blake (1994) and Loftus and Ruthruff (1994). In their view, metamers are physically different objects that in well-defined circumstances elicit identical responses or judgments. With a change of circumstances, the physically different objects are responded to or judged accordingly. The contrast supports the assertion that the hierarchy of metamers suggested by Loftus and Ruthruff (1994) and Backus (2002) should be expanded to include a variety of cognitive metamers, most notably, with intention as the metamer-breaking operation. The contrast raises the question of whether cognitive metamers, with intention as the metamer-breaking operation, should be added to the hierarchy of metamers suggested by Loftus and Ruthruff (1994) and Backus (2002).

In past experimental situations where the participant has been asked to judge weight through lifting, hefting, or wielding, neither the physical quantity nor the psychological state may have been appropriately characterized. The present research raises the possibilities that (1) instead of weight, the physical characterization is a point in the mass $\times$ ellipsoid volume $\times$ ellipsoid symmetry space, and (2) instead of heaviness, the psychological state is moveableness (or maneuverability or controllability) - most likely, moveableness in a particular way. Mass as the relevant dimension in Figure 1 rather than weight (mass $\times$ gravitational acceleration) means that the data patterns of Experiments $1-4$ should be indifferent to gravity. That is, the metamers and nonmetamers of moveableness should be invariant over 0,1 , and $2 \mathrm{G}$, for example.

A further implication of the space depicted in Figure 1 is that neither the mapping of one weight value to multiple perceptions nor the mapping of multiple weight values to the same perception may require an interpretation in terms of subjectivism or illusion (Turvey et al., 2001). Cross and Rotkin (1975) identified important parallels between the facts of heaviness perception and those of other perceptual phenomena. In particular, they noted the similarities between heaviness perception and loudness perception. As is well known, the latter depends on tonal frequency as well as intensity. Loudness metamers in the form of equal-loudness contours in a coordinate space of sound pressure and frequency suggested to Cross and Rotkin the possibility of constructing equal-heaviness contours in the coordinate space of weight and size. They underscored the degree of similarity of the contours in the two spaces. The slopes of equal-loudness contours increase as the level of frequency decreases. Similarly, the slopes of equal-heaviness contours increase as size level decreases (Cross \& Rotkin, 1975; Stevens \& Rubin, 1970). The facts of loudness perception are attributed to the operating characteristics of the auditory system and are not discussed as illusory in textbooks on hearing (e.g., Han- del, 1993). As observed by Cross and Rotkin the facts of heaviness perception should be addressed analogously. That is, they should be viewed as reflecting the operating characteristics of dynamic touch.

By way of summary, it is worthwhile to consider that experimental psychology's long-term puzzlement over weight perception has been sustained by the elusiveness of the "natural kinds"- - the objective properties or kinds that could support a generalization of facts about weight perception. Arguably, the natural kinds have been elusive because their discovery requires joint consideration of the laws of rigid body motion, the proper function of dynamic touch, and the objective status of relational properties.

A proper function of a perceptual system is a function that it has executed successfully in the history of one or more species to warrant reproduction (Millikan, 1984, 1993). The manifest ability to control the movements of limbs and hand-held objects suggests a link between the persistent perceptual achievements of the human movement system and its persistent biomechanical achievements. The understanding of both the perceptual and motoric achievements must be grounded in the laws of rigid body translation (constrained by mass) and rotation (constrained by the inertia tensor). A reasonable guess at the proper perceptual function of dynamic touch, therefore, is attunement to the motion laws. In particular, it is attunement to the laws' parameters and the relation between them in a manner that bears, as noted earlier, on the neuromuscular challenge of producing the requisite forces for translation and rotation (Shockley et al., 2001; Turvey et al., 1999; Turvey et al., 2001). If the guess is right, then a rationalization is provided for regarding perception of heaviness as, more aptly, the perception of moveableness.

A full understanding of the perception of moveableness requires recognizing that the real, objective, and physical properties underlying the perception are, as Gibson (1979) and Bunge (1977) might say, possessed by the animalobject interface rather than by either the animal or the object separately. The term affordance has been adopted for such properties (Gibson, 1979; Turvey, 1992). Moveableness is the perception of the opportunities a hand-held object affords for varying the patterns and levels of muscular forces by which the object can be moved in a controlled fashion. A hand-held object is hard to move or to maneuver to the extent that there are relatively few ways in which forces can be applied to the object in order to bring about a desired trajectory. The objective, real, and physical basis for this affordance of moveableness is the hand-held object's mass and mass distribution taken with reference to the force-producing neuromuscular system. In this complementation of object and neuromuscular system, the volume and symmetry of the inertia tensor emerge as relevant physical quantities.

Finally, we note that it is currently unclear why the physical quantities combine in the specific proportions illustrated in Figure 1. Future investigations of how each of these constraints on an object's maneuverability is manifest perceptually may provide some insight into this prob- 
lem. For example, changing the capacity for generating forces (by wielding about the elbow, shoulder, or with more than one hand) should influence how difficult it feels to move an object (quantified by ellipsoid volume) without changing the actual pattern of forces required to move the object in the desired manner (quantified by ellipsoid symmetry). Furthermore, as implied above, if the proposed inertial constraints are appropriate for evaluating perception of heaviness/moveableness, then similar studies performed in different gravitational fields should yield quantitatively comparable results.

\section{REFERENCES}

AmazeEn, E. (1997). The effects of volume on perceived heaviness by dynamic touch: With and without vision. Ecological Psychology, 9, 245-263.

Amazeen, E. (1999). Perceptual independence of size and weight by dynamic touch. Journal of Experimental Psychology: Human Perception \& Performance, 25, 102-119.

AMAZEEN, E., \& TuRvEY, M. T. (1996). Weight perception and the haptic size-weight illusion are functions of the inertia tensor. Journal of Experimental Psychology: Human Perception \& Performance, 22, 213-232.

ANDERSON, N. H. (1970). Averaging model applied to the size-weight illusion. Perception \& Psychophysics, 8, 1-4.

ARNOLD, V. I. (1989). Mathematical methods of classical mechanics. New York: Springer-Verlag.

Ashby, G., \& Townsend, J. (1986). Varieties of perceptual independence. Psychological Review, 93, 154-179.

Backus, B. T. (2002). Perceptual metamers in stereoscopic vision. In T. G. Dietterich, S. Becker, \& Z. Ghahramani (Eds.), Advances in neural information processing systems: Vol. 14. Cambridge, MA: MIT Press.

BeLL, C. (1826). On the nervous circle which connects the voluntary muscles with the brain. Philosophical Transactions of the Royal Society of London, 116, 163-167.

BORISENKO, A. I., \& TAPAROV, I. E. (1979). Vector and tensor analysis. New York: Dover.

Bunge, M. (1977). Treatise on basic philosophy (Vol. 3). Boston: Reidel.

Charpentier, A. (1891). Analyse experimentale de quelques elements de la sensation de poids [Experimental study of some aspects of weight perception]. Archives de Physiologie Normales et Pathologiques, 3, 122-135.

Clark, A. (2000). A theory of sentience. Oxford: Oxford University Press.

Cross, D. V., \& Rotkin, L. (1975). The relation between size and apparent heaviness. Perception \& Psychophysics, 18, 79-87.

Davis, C. M., \& BricketT, P. (1977). The role of preparatory muscle tension in the size-weight illusion. Perception \& Psychophysics, 22, 262-264.

Davis, C. M., \& RoberTs, W. (1976). Lifting movements in the sizeweight illusion. Perception \& Psychophysics, 20, 33-36.

Den Hartog, J. P. (1961). Mechanics. New York: Dover.

DRETSKe, F. (1995). Naturalizing the mind Cambridge, MA: MIT Press.

Ellis, R. R., \& Lederman, S. (1993). The role of haptic versus visual volume cues in the size-weight illusion. Perception \& Psychophysics, 53, 315-324.

FESTA, E., \& WELCH, L. (1998). Perceived speed of motion metamers depend on component signal strength [Abstract]. Investigative Opthalmology \& Visual Science, 39(Suppl.), 1983.

GiBson, J. J. (1966). The senses considered as perceptual systems. Boston: Houghton Mifflin.

Gibson, J. J. (1979). The ecological approach to visual perception. Boston: Houghton Mifflin.

Gordon, A. M., Forssberg, R. S., Johansson, R. S., \& Westling, G. (1991). Visual size cues in the programming of manipulative forces during precision grip. Experimental Brain Research, 83, 477-482.
GRANIT, R. (1972). Constant errors in the execution and appreciation of movement. Brain, 95, 649-660.

Gregory, R. L. (1974). Concept and mechanisms of perception. New York: Scribner.

HANDEL, S. (1993). Listening. Cambridge, MA: MIT Press.

HARDIN, C. L. (1993). Color for philosophers. Indianapolis: Hackett.

Hilbert, D. (1992). What is color vision? Philosophical Studies, 68, 351-370.

Jones, L. A. (1986). Perception of force and weight: Theory and research. Psychological Bulletin, 100, 29-42.

KibBLe, T. W. B. (1985). Classical mechanics. London: Longman.

Kingma, I., Beek, P., \& van DieEn, J. H. (2002). The inertia tensor versus static moment and mass in perceiving length and heaviness of hand-wielded rods. Journal of Experimental Psychology: Human Perception \& Performance, 28, 180-191.

Kingma, I., Salversbergh, G. J. P., \& Tousaint, H. M. (1999). Object size effects on initial lifting forces under microgravity conditions. $E x$ perimental Brain Research, 124, 422-428.

Kreidfeldt, J. G., \& ChuanG, M.-C. (1979). Moment of inertia: Psychophysical study of an overlooked sensation. Science, 206, 588-590.

LoFTUS, G., \& RUTHRUFF, E. (1994). A theory of visual information acquisition and visual memory with special application to intensityduration tradeoffs. Journal of Experimental Psychology: Human Perception \& Performance, 20, 33-49.

Millikan, R. (1984). Language, thought and other biological categories. Cambridge, MA: MIT Press.

Millikan, R. (1993). White queen psychology and other essays for Alice. Cambridge, MA: MIT Press.

PAYNe, B., \& DaVIS, R. C. (1940). The role of muscular tension in the comparison of lifted weights. Journal of Experimental Psychology, 27, 227-242.

RichaRds, W., \& RiLEY, M. (1977). Texture metamers. Journal of the Optical Society of America, 67, 1401A.

Ross, H. E. (1969). When is weight not illusory? Quarterly Journal of Experimental Psychology, 21, 346-355.

Ross, H. E., \& GrEgory, R. L. (1970). Weight illusions and weight discrimination-A revised hypothesis. Quarterly Journal of Experimental Psychology, 22, 318-328.

Ross, P. W. (2001). The location problem for color subjectivism. Cognition \& Consciousness, 10, 42-58.

SeKuler, R., \& Blake, R. (1994). Perception (3rd ed.). New York: McGraw-Hill.

Shockley, K., Grocki, M., Carello, C., \& Turvey, M. T. (2001). Somatosensory attunement to the rigid body laws. Experimental Brain Research, 136, 133-137.

SJöBERG, L. (1969). Sensation scales in the size-weight illusion. Scandinavian Journal of Psychology, 10, 109-112.

Solomon, H. Y., \& TurveY, M. T. (1988). Haptically perceiving the distances reachable with hand-held objects. Journal of Experimental Psychology: Human Perception \& Performance, 14, 404-427.

Stevens, J. C., \& Rubin, L. L. (1970). Psychophysical scales of apparent heaviness and the size-weight illusion. Perception \& Psychophysics, 8, 225-230.

Stroop, M., Turvey, M. T., Fitzpatrick, P., \& Carello, C. (2000). Inertia tensor and weight-percept models of length perception by static holding. Journal of Experimental Psychology: Human Perception \& Performance, 26, 1133-1147.

Treue, S., Hol, K., \& Rauber, E. J. (2000). Seeing multiple directions of motion - psychophysics and physiology. Nature Neuroscience, $\underline{\mathbf{3}}_{2}$ 270-276.

TURVEY, M. T. (1992). Affordances and prospective control: An outline of ontology. Ecological Psychology, 4, 173-188.

Turvey, M. T., Burton, G., Amazeen, E. L., Butwill, M., \& Carello, C. (1998). Perceiving the width and height of a hand-held object by dynamic touch. Journal of Experimental Psychology: Human Perception \& Performance, 24, 35-48.

Turvey, M. T., Shockley, K., \& CARELlo, C. (1999). Affordance, proper function and the physical basis of perceived heaviness. Cognition, 73, B17-B26.

Turvey, M. T., Whitmyer, V., \& Shockley, K. (2001). Explaining 
metamers: Right degrees of freedom, not subjectivism. Consciousness \& Cognition, 10, 105-116.

WEBER, E. H. (1978). The sense of touch (H. E. Ross, Ed. \& Trans.). London: Academic Press. (Original work published 1834)

Williams, D., Tweten, S., \& SeKuler, R. (1991). Using metamers to explore motion perception. Vision Research, 31, 275-286.

ZEKI, S. (1983). Colour coding in the cerebral cortex: The reaction of cells in monkey visual cortex to wavelengths and colours. Neuroscience, 9, 741-765.

\section{NOTES}

1. The major benefit of developing the relevant quantities in terms of the inertia ellipsoid (see Figure 2) is that, in the general physical case, the inertia ellipsoid completely determines the rotational characteristics of an object (e.g., Arnold, 1989). Two objects with identical inertia ellipsoids will exhibit identical rotational motions for identical initial conditions - regardless of differences in their shapes, linear dimensions, material compositions, weights, and densities. The small conceptual cost of focusing on the ellipsoid is that the links between an object and the parameters of its inertia ellipsoid are not always intuitive.

The ellipsoid for any given object is generated by passing all possible axes $p$ through the rotation point $O$ with lengths $O A$ laid off on each axis with magnitudes equal to $\left(I_{p}\right)^{-1 / 2}$. The loci of $A$ comprise the ellipsoid, and the principal axes of the ellipsoid coincide with the principal axes of inertia $\mathbf{e}_{k}$ of the object (e.g., Arnold, 1989; Borisenko \& Taparov, 1979). The ellipsoid bears a crude resemblance to the shape of a rigid object (Arnold, 1989; Turvey, Burton, Amazeen, Butwill, \& Carello, 1998). If an object is stretched out along some axis $\mathbf{e}_{i}$ (consider a rectangular parallelepiped that is longer than it is wide), then the moment of inertia $I_{i}$ with respect to this axis is small, and in consequence the inertia ellipsoid is also stretched out along this axis. In contrast, the relation between mean rotational inertia and ellipsoid volume is counterintuitive. The two quantities are inversely related: Larger mean resistance is associated with smaller ellipsoid volume. An additional drawback of ellipsoid volume is the unit $\left(\mathrm{kg} \cdot \mathrm{m}^{2}\right)^{-3 / 2}$. The tensor's determinant (product of eigenvalues) and trace (sum of eigenvalues) provide simpler measures of an object's total resistance to rotation, but they are not descriptors of the ellipsoid.

2. Computations of the inertia tensor, ellipsoid volume, and ellipsoid symmetry were done by means of the Inertia Tensor Calculation Graphical User Interface (K. Shockley, University of Connecticut) in the Matlab (Mathworks Inc.) programming environment. This software permits the user to manipulate a tensor object's mass distribution graphically, while providing on-line calculations and schematic representations of its diagonalized tensor and inertia ellipsoid. The calculations of tensor components

$$
\begin{array}{ccc}
I_{X X} & I_{X Y} & I_{X Z} \\
I_{Y X} & I_{Y Y} & I_{Y Z} \\
I_{Z X} & I_{Z Y} & I_{Z Z}
\end{array}
$$

were based on the parallel axis theorems for moments and products of inertia (e.g., Den Hartog, 1961; Kibble, 1985; see also Stroop, Turvey, Fitzpatrick, \& Carello, 2000). Because $O$ was not on any given rod's central symmetry axis, there was always at least one nonzero product of inertia (one off-diagonal term in the inertia tensor matrix). Diagonalization of the tensors was achieved using the Matlab function for deriving eigenvalues and eigenvectors.

3 . The regression equation that has been reported elsewhere $[$ perceived heaviness $=113.78+.15$ mass -31.17 ellipsoid volume -63.76 ellipsoid symmetry (Shockley et al., 2001; Turvey et al., 2001)] did not use standard units for mass or the eigenvalues on which ellipsoid volume and symmetry are based. We elected, instead, to use those units that would result in numbers that were manageable in tabular form. Using a consistent $\mathrm{kg} \cdot \mathrm{m}$ system produces identical dependencies between perceived heaviness and $M, S$, and $V$ with respect to the standard coefficients, $t$ and $p$ values.

4. Although these objects and those in subsequent experiments are light compared with those that formed the basis of Weber's law, it must be emphasized that weight per se is not the limiting factor on wielding. While a kilo is wieldable in some configurations, our stimuli actually already push the limits of what female undergraduates can comfortably wield. For comparison, a bottle of juice weighing $1.70 \mathrm{~kg}$ is easier to move than many of our objects due to its compactness, where it is typically grasped, and how it is typically moved.

5. Experiments have shown that haptic sensitivity to the inertial parameters is independent of the forcefulness of wielding. For example, the perceptual effects of rotational inertia are constant over variations in mean torque levels brought about by experimenter-imposed restrictions on angular acceleration (Amazeen \& Turvey, 1996; Solomon \& Turvey, 1988).

(Manuscript received July 9, 2001; revision accepted for publication October 5, 2003.) 